PROCEEDINGS OF THE

AMERICAN MATHEMATICAL SOCIETY

Volume 133, Number 10, Pages 2897-2902

S 0002-9939(05)08071-8

Article electronically published on April 25, 2005

\title{
THE POLYNOMIAL ANALOGUE OF A THEOREM OF RÉNYI
}

\author{
KENT E. MORRISON
}

(Communicated by David E. Rohrlich)

\begin{abstract}
Rényi's result on the density of integers whose prime factorizations have excess multiplicity has an analogue for polynomials over a finite field.
\end{abstract}

Let $n=p_{1}^{\alpha_{1}} \cdots p_{r}^{\alpha_{r}}$ be the prime factorization of a positive integer $n$. Define the excess of $n$ to be $\left(\alpha_{1}-1\right)+\cdots+\left(\alpha_{r}-1\right)$, which is the difference between the total multiplicity $\alpha_{1}+\cdots+\alpha_{r}$ and the number of distinct primes in the factorization. An integer with excess 0 is also said to be square-free. Let $E_{k}$ denote the set of positive integers of excess $k, k=0,1,2, \ldots$ Rényi proved that the set $E_{k}$ has a density $d_{k}$ and that the sequence $\left\{d_{k}\right\}$ has a generating function given by

$$
\sum_{k \geq 0} d_{k} z^{k}=\prod_{p}\left(1-\frac{1}{p}\right)\left(1+\frac{1}{p-z}\right),
$$

where the product extends over the primes. Recall that the density of a set of positive integers $E$ is the limit (if it exists)

$$
\lim _{n \rightarrow \infty} \frac{\#(E \cap\{1,2, \ldots, n\})}{n},
$$

which is the limiting probability that an integer from 1 to $n$ is in $E$.

The set of square-free integers is $E_{0}$ and setting $z=0$ in the generating function gives $d_{0}=\prod_{p}\left(1-1 / p^{2}\right)$, which is the well-known result that the density of squarefree integers is $1 / \zeta(2)=6 / \pi^{2}$. (This was first proved by Gegenbauer [2] in 1885. A clear, non-rigorous presentation is in [3].) By setting $z=1$ one sees that $\sum_{k} d_{k}=1$, so that the density is countably additive on the specific partition of $\mathbf{Z}^{+}$given by the $E_{k}$. Rényi's proof appeared in [7], but an alternative proof was given by Kac in [4, pp. 64-71].

The aim of this paper is to derive an analogue of the generating function for polynomials in one variable over a finite field. Let $\mathbf{F}_{q}$ be the field with $q$ elements and let $\mathbf{F}_{q}[x]$ be the polynomial ring. The prime elements of $\mathbf{F}_{q}[x]$ are the irreducible monic polynomials. Let $f$ be a monic polynomial with prime factorization $f=$ $\pi_{1}^{\alpha_{1}} \cdots \pi_{r}^{\alpha_{r}}$, and define the excess of $f$ to be $\left(\alpha_{1}-1\right)+\cdots+\left(\alpha_{r}-1\right)$, just as for an integer. Let $e_{n, k}$ be the number of monic polynomials of degree $n$ and excess $k$. Define

$$
d_{n, k}=\frac{e_{n, k}}{q^{n}}
$$

Received by the editors June 9, 2004.

2000 Mathematics Subject Classification. Primary 11T06; Secondary 11T55, 05A16.

(C)2005 American Mathematical Society

Reverts to public domain 28 years from publication 
which is the probability that a monic polynomial of degree $n$ has excess $k$. Note that $d_{0, k}=0$ for $k>0$. Then define the analogue of the density to be the limiting "probability" as the degree goes to infinity:

$$
d_{k}=\lim _{n \rightarrow \infty} d_{n, k} .
$$

Define $D(z)=\sum_{k \geq 0} d_{k} z^{k}$ to be the ordinary power series generating function of the sequence $\left\{d_{k}\right\}$. Let $\mathrm{N} f=q^{\operatorname{deg} f}$ be the norm of the polynomial $f$, which is the cardinality of the residue ring $\mathbf{F}_{q}[x] /(f)$. The main result of this paper is the following theorem concerning $D(z)$.

Theorem 1. The generating function $D(z)$ has a factorization over the prime polynomials given by

$$
D(z)=\prod_{\pi}\left(1-\frac{1}{\mathrm{~N} \pi}\right)\left(1+\frac{1}{\mathrm{~N} \pi-z}\right) .
$$

Proof. We begin with the geometric series

$$
\frac{1}{1-q t}=\sum_{n \geq 0} q^{n} t^{n}
$$

which is the generating function for the number of monic polynomials of degree $n$. Then unique factorization in $\mathbf{F}_{q}[x]$ allows us to factor the generating function formally:

$$
\begin{aligned}
\frac{1}{1-q t} & =\prod_{\pi} \sum_{j \geq 0} t^{j \operatorname{deg} \pi} \\
& =\prod_{\pi} \frac{1}{1-t^{\operatorname{deg} \pi}} .
\end{aligned}
$$

By grouping the primes of the same degree and letting $\nu_{i}$ denote the number of primes of degree $i$, we can rewrite the last line above as

$$
\frac{1}{1-q t}=\prod_{i \geq 1}\left(\frac{1}{1-t^{i}}\right)^{\nu_{i}} \text {. }
$$

From this it follows that

$$
1-q t=\prod_{i \geq 1}\left(1-t^{i}\right)^{\nu_{i}}
$$

as a formal power series. In the product on the right there is a finite number of terms for each power of $t$ so that the coefficients make sense. In fact, the coefficient of $t^{n}$ is 0 except for $n=0,1$. However, considered as a function of a complex variable $t$, the product does not converge for all $t$. It does converges absolutely for $|t|<1 / q$. This follows from consideration of the series $\sum \nu_{i} t^{i}$ and the fact that $\nu_{i}$ is asymptotic to $q^{i} / i$.

Next we define the two-variable generating function

$$
E(t, z)=\sum_{n, k} e_{n, k} t^{n} z^{k} .
$$

Modifying the factorization in (11), we see that

$$
E(t, z)=\prod_{\pi}\left(1+t^{\operatorname{deg} \pi}+t^{2 \operatorname{deg} \pi} z+\cdots+t^{j \operatorname{deg} \pi} z^{j-1}+\cdots\right) .
$$


Note that the variable $z$ appears with a power that is equal to the excess multiplicity. That is, if $f=\pi_{1}^{\alpha_{1}} \cdots \pi_{r}^{\alpha_{r}}$, then the product expansion of $E(t, z)$ has a term of the form $t^{\alpha_{1} \operatorname{deg} \pi_{1}} \cdots t^{\alpha_{r} \operatorname{deg} \pi_{r}} z^{\alpha_{1}-1} \cdots z^{\alpha_{r}-1}$. Sum the geometric series in each factor to obtain the formal factorization

$$
E(t, z)=\prod_{\pi}\left(1+\frac{t^{\operatorname{deg} \pi}}{1-t^{\operatorname{deg} \pi} z}\right) .
$$

Group the irreducibles by degree to get

$$
E(t, z)=\prod_{i}\left(1+\frac{t^{i}}{1-t^{i} z}\right)^{\nu_{i}}
$$

Now the product on the right converges absolutely if and only if the series

$$
\sum_{i \geq 1} \nu_{i}\left|\frac{t^{i}}{1-t^{i} z}\right|
$$

converges. We claim that (4) converges for $|t|<1 / q$ and $|z|<q$, because the denominators $\left|1-t^{i} z\right|$ are bounded away from 0 and $\nu_{i}$ is asymptotic to $q^{i} / i$. (Actually, it suffices that $\nu_{i}<q^{i}$.)

From (21) and (31) we get

$$
(1-q t) E(t, z)=\prod_{i \geq 1}\left(1-t^{i}\right)^{\nu_{i}} \prod_{i \geq 1}\left(1+\frac{t^{i}}{1-t^{i} z}\right)^{\nu_{i}} .
$$

On the domain where both products converge absolutely, we can combine the factors for each $i$ to get

$$
(1-q t) E(t, z)=\prod_{i \geq 1}\left(1-t^{i}\right)^{\nu_{i}}\left(1+\frac{t^{i}}{1-t^{i} z}\right)^{\nu_{i}} .
$$

By multiplying the factors together we can see that the absolute convergence of the infinite product depends on the convergence of the series

$$
\sum_{i} \nu_{i}\left|\frac{t^{2 i} z-t^{2 i}}{1-t^{i} z}\right|
$$

Then reasoning along the same lines as before, we see that this series converges for $\left|t^{2}\right|<q$ and $|z|<\sqrt{q}$. In particular, the product converges for $t=1 / q$, and so after carrying out the multiplication of the left side of (15) we arrive at

$$
\sum_{n, k}\left(e_{n, k}-q e_{n-1, k}\right) t^{n} z^{k}=\prod_{i \geq 1}\left(1-t^{i}\right)^{\nu_{i}}\left(1+\frac{t^{i}}{1-t^{i} z}\right)^{\nu_{i}} .
$$

We evaluate this at $t=1 / q$ to get

$$
\sum_{n, k}\left(e_{n, k}-q e_{n-1, k}\right)(1 / q)^{n} z^{k}=\prod_{i \geq 1}\left(1-(1 / q)^{i}\right)^{\nu_{i}}\left(1+\frac{(1 / q)^{i}}{1-(1 / q)^{i} z}\right)^{\nu_{i}} .
$$

The coefficient of $z^{k}$ is the sum $\sum_{n \geq 1}\left(e_{n, k} / q^{n}-e_{n-1, k} / q^{n-1}\right)$. This telescopes to give

$$
\lim _{n \rightarrow \infty} \frac{e_{n, k}}{q^{n}}=\lim _{n \rightarrow \infty} d_{n, k}
$$


which is the definition of $d_{k}$, and so we have

$$
D(z)=\sum_{k} d_{k} z^{k}=\prod_{i \geq 1}\left(1-(1 / q)^{i}\right)^{\nu_{i}}\left(1+\frac{(1 / q)^{i}}{1-(1 / q)^{i} z}\right)^{\nu_{i}} .
$$

Finally, we write the product by indexing over the prime polynomials $\pi$ and note that the norm of $\pi$ is $\mathrm{N} \pi=q^{\operatorname{deg} \pi}$. With this we have the generating function for $d_{k}$ in the form that is most directly analogous to Rényi's theorem:

$$
\begin{aligned}
D(z) & =\prod_{\pi}\left(1-(1 / q)^{\operatorname{deg} \pi}\right)\left(1+\frac{(1 / q)^{\operatorname{deg} \pi}}{1-(1 / q)^{\operatorname{deg} \pi} z}\right) \\
& =\prod_{\pi}\left(1-\frac{1}{\mathrm{~N} \pi}\right)\left(1+\frac{1}{\mathrm{~N} \pi-z}\right) .
\end{aligned}
$$

The coefficient $d_{0}$ is the limiting "probability" that a monic polynomial is squarefree. To develop the analogy with the density of the square-free integers given by $d_{0}$ in Rényi's generating function, we use the zeta function of $\mathbf{F}_{q}[x]$ (i.e. the zeta function of the affine line over $\mathbf{F}_{q}$ )

$$
\zeta(s)=\frac{1}{1-q^{-s}}
$$

which immediately comes from the definition

$$
\zeta(s)=\sum_{\mathfrak{a}} \frac{1}{(\mathrm{Na})^{s}},
$$

where the sum is over all ideals of $\mathbf{F}_{q}[x]$ and the norm $\mathrm{Na}$ is the cardinality of the residue ring $\mathbf{F}_{q}[x] / \mathfrak{a}$. It has a factorization over the prime ideals (i.e. irreducible polynomials)

$$
\begin{aligned}
\zeta(s) & =\prod_{\pi} \frac{1}{1-(\mathrm{N} \pi)^{-s}} \\
& =\prod_{i \geq 1}\left(\frac{1}{1-q^{-i s}}\right)^{\nu_{i}} .
\end{aligned}
$$

Corollary 1. $d_{0}=\frac{1}{\zeta(2)}=1-\frac{1}{q}$.

Proof. We have

$$
d_{0}=D(0)=\prod_{i \geq 1}\left(1-\frac{1}{q^{2 i}}\right)^{\nu_{i}} .
$$

Then in (2) we may let $t=1 / q^{2}$, because the product converges for $|t|<1 / q$, to obtain

$$
1-\frac{1}{q}=\prod_{i \geq 1}\left(1-\frac{1}{q^{2 i}}\right)^{\nu_{i}}
$$

Note that the product is $1 / \zeta(2)$.

Corollary 1 can be obtained as a special case of much more general results on square-free values of polynomials in one or more variables from the work of Ramsay [6] and Poonen [5]. It turns out that for $n \geq 2$, the value of $d_{n, 0}$ is $1-1 / q$. This can 
be seen by finding the coefficients $e_{n, 0}$, which count the number of monic, squarefree polynomials of degree $n$. These polynomials can be counted directly; see, for example, [1].

Corollary 2. The number of square-free monic polynomials of degree $n \geq 2$ is $q^{n}-q^{n-1}$.

Proof. The generating function $\sum_{n \geq 0} e_{n, 0} t^{n}=E(t, 0)$. From (3) we see that

$$
E(t, 0)=\prod_{i \geq 1}\left(1+t^{i}\right)^{\nu_{i}}
$$

Using (2) we see that

$$
\begin{aligned}
E(t, 0)(1-q t) & =\prod_{i \geq 1}\left(1+t^{i}\right)^{\nu_{i}}\left(1-t^{i}\right)^{\nu_{i}} \\
& =\prod_{i \geq 1}\left(1-t^{2 i}\right)^{\nu_{i}} \\
& =1-q t^{2} .
\end{aligned}
$$

Therefore,

$$
E(t, 0)=\frac{1-q t^{2}}{1-q t}
$$

from which it follows that $e_{n, 0}=q^{n}-q^{n-1}$ for $n \geq 2$.

From the expression

$$
D(z)=\prod_{i \geq 1}\left(1-\frac{1}{q^{i}}\right)^{\nu_{i}}\left(1+\frac{1}{q^{i}-z}\right)^{\nu_{i}}
$$

we can see that $D(z)$ has poles at $z=q^{i}$ of multiplicity $\nu_{i}$. In particular the pole at $z=q$ has multiplicity $q-1$. Elementary analysis of the singularity there, along the lines of Kac [4 in his discussion of Rényi's result, enables us to describe the asymptotic behavior of the $d_{k}$ as $k \rightarrow \infty$.

Corollary 3. As $k$ goes to infinity, $d_{k}$ is asymptotic to

$$
A \frac{k^{q-2}}{q^{k}}
$$

where the constant $A$ is given by

$$
A=\frac{1}{(q-2) !}\left(\frac{1}{q}-\frac{1}{q^{2}}\right)^{q-1} \prod_{i \geq 2}\left(1-\frac{1}{q^{i}}\right)^{\nu_{i}}\left(1-\frac{1}{q^{i}-q}\right)^{\nu_{i}} .
$$

One may contrast this asymptotic result with the classical case of Rényi. Although the generating functions have clearly analogous form, the generating function for the number-theoretic version has only a simple pole $z=2$, which is the pole of smallest absolute value. The asymptotic analysis shows that

where

$$
d_{k} \sim \frac{\delta}{2^{k}}
$$

$$
\delta=\frac{1}{4} \prod_{p \geq 3} \frac{(p-1)^{2}}{p(p-2)}
$$


The referee has observed that Theorem 1 of this article can be extended naturally to function fields over finite fields by using $S$-zeta functions and their residues at $t=1 / q$. Let $K$ be a function field over the constant field $\mathbf{F}_{q}$. Let $S$ be a finite, non-empty set of places on $K$ and let $\mathcal{O}_{K, S}$ denote the ring of $S$-integers of $K$. Then for every integer $k \geq 0$, the density $d_{k, S}$ of ideals in $\mathcal{O}_{K, S}$ with excess $k$ exists, and the following analytic identity holds:

$$
\sum_{k \geq 0} d_{k} z^{k}=\prod_{v \notin S}\left(1-\frac{1}{\mathrm{~N} v}\right)\left(1+\frac{1}{\mathrm{~N} v-z}\right),
$$

where for each place $v$ on $K$ the norm $\mathrm{N} v=q^{\operatorname{deg} v}$ is the cardinality of the residue field at $v$.

Finally, the referee has pointed out that by generalizing Kac's proof of Rényi's theorem [4, pp. 64-71], there should also be an analogue of the theorem for the density of ideals with excess $k$ in the ring of algebraic integers (or ring of $S$-integers) of any number field.

\section{REFERENCES}

[1] L. Carlitz. An application of a theorem of Stickelberger, Simon Stevin 31 (1956) 27-30. MR0080696 (18:285g)

[2] L. Gegenbauer. Asymptotische Gesetze der Zahlentheorie, Denkshcriften Akad. Wien 49 (1885) 37-80

[3] G. A. Jones. 6/ $\pi^{2}$, Mathematics Magazine 66 (1993) 290-298. MR1251442 (94m:11002)

[4] M. Kac. Statistical Independence in Probability, Analysis and Number Theory. Carus Monographs, no. 12. Mathematical Association of America, Washington, D.C., 1959. MR0110114 $(22: 996)$

[5] B. Poonen. Squarefree values of multivariable polynomials, Duke Math. J. 118 (2003), no. 2, 353-373. MR1980998 (2004d:11094)

[6] K. Ramsay. Square-free values of polynomials in one variable over function fields, Internat. Math. Res. Notices, no. 4 (1992) 97-102. MR.1159451 (93b:11115)

[7] A. Rényi. On the density of certain sequences of integers, Acad. Serbe Sci. Publ. Inst. Math. 8 (1955), 157-162. MR0076787|(17,944f)

Department of Mathematics, California Polytechnic State University, San Luis OBispo, CALifornia 93407

E-mail address: kmorriso@calpoly.edu 